\title{
Pseudoaneurysm Formation due to Popliteal Artery Injury Caused by Drilling during Medial Opening Wedge High Tibial Osteotomy
}

\author{
Keun Churl Chun, $\mathrm{MD}^{1,2}$, Byung Jun So, $\mathrm{MD}^{3}$, Hyun Tak Kang, $\mathrm{MD}^{1}$, and Churl-Hong Chun, MD, $\mathrm{PhD}^{1}$ \\ ${ }^{1}$ Department of Orthopedic Surgery, Wonkwang University College of Medicine, Iksan; ${ }^{2}$ Department of Orthopedic Surgery, Mokpo Hankook Hospital, Mokpo; \\ ${ }^{3}$ Department of Vascular Surgery, Wonkwang University College of Medicine, Iksan, Korea
}

\begin{abstract}
We report a case of 53-year-old woman with an injured popliteal artery due to excessive drilling with a drill bit during medial opening wedge high tibial osteotomy (MOWHTO). Pseudoaneurysm was diagnosed three days after surgery and confirmed by urgent computed tomography (CT) angiography. Open vascular surgery with resection of the perivascular hematoma and end-to-end anastomosis using ipsilateral saphenous vein interposition graft was performed. CT angiography at 8 months postoperatively showed that blood flow was maintained without obstruction of the graft site and active dorsiflexion of the foot was possible. To reduce neurovascular injury during MOWHTO, it is important not to drill the far cortex at the proximal part of the osteotomy site when using a drill bit, and the metal should be positioned posteromedially as much as possible.
\end{abstract}

Keywords: Knee, Osteotomy, Popliteal artery, Pseudoaneurysm

Medial opening wedge high tibial osteotomy (MOWHTO) is an effective method for the treatment of osteoarthritis confined to the medial side of the knee joint with varus axial malalignment. We experienced a case of pseudoaneurysm of the popliteal artery after MOWHTO inflicted by a drill bit. To our knowledge, there has been no report of injury of the popliteal artery due to drilling with a drill bit during MOWHTO for screw fixation of the locking plate. We postulate the mechanism of this vascular injury and describe the clinical features and management.

Received June 13, 2018; Revised August 9, 2018;

Accepted August 10, 2018

Correspondence to: Churl-Hong Chun, $\mathrm{MD}, \mathrm{PhD}$

Department of Orthopedic Surgery, School of Medicine, Wonkwang University, 895 Muwang-ro, Iksan 54538, Korea

Tel: +82-63-859-1360, Fax: +82-63-852-9329

E-mail: cch@wku.ac.kr

Source of funding: This study was supported by a Wonkwang University research grant in 2018 .

This is an Open Access article distributed under the terms of the Creative Commons Attribution Non-Commercial License (http://creativecommons.org/licenses/by-nc/4.0/) which permits unrestricted non-commercial use, distribution, and reproduction in any medium, provided the original work is properly cited.

\section{Case Report}

A 53-year-old female patient underwent MOWHTO for medial compartment osteoarthritis. A preoperative radiograph showed degenerative osteoarthritis with medial joint space narrowing, sclerosis, and $6^{\circ}$ of varus deformity. Magnetic resonance imaging (MRI) showed unicompartmental osteoarthritis of the medial femoral condyle and medial tibial condyle corresponding to Outerbridge grade IV; therefore, MOWHTO was planned. After confirming there was no instability of the ligaments under spinal anesthesia, the cartilage and the meniscus were assessed through arthroscopy. Arthroscopy revealed cartilage lesions in the medial femoral condyle and medial tibial condyle, and microfracture was performed (Fig. 1A).

After the arthroscopic procedure, a longitudinal skin incision of about $8 \mathrm{~cm}$ was performed on the medial side of the knee joint. The pes anserinus was exposed and pulled downward, and the superficial medial collateral ligament was subperiosteally released from the tibia, and the Hohmann retractor was inserted into the posterior medial side of the tibia to protect the neurovascular structures and expose the osteotomy site.

Under fluoroscopic guidance, the Kirschner wire was inserted 

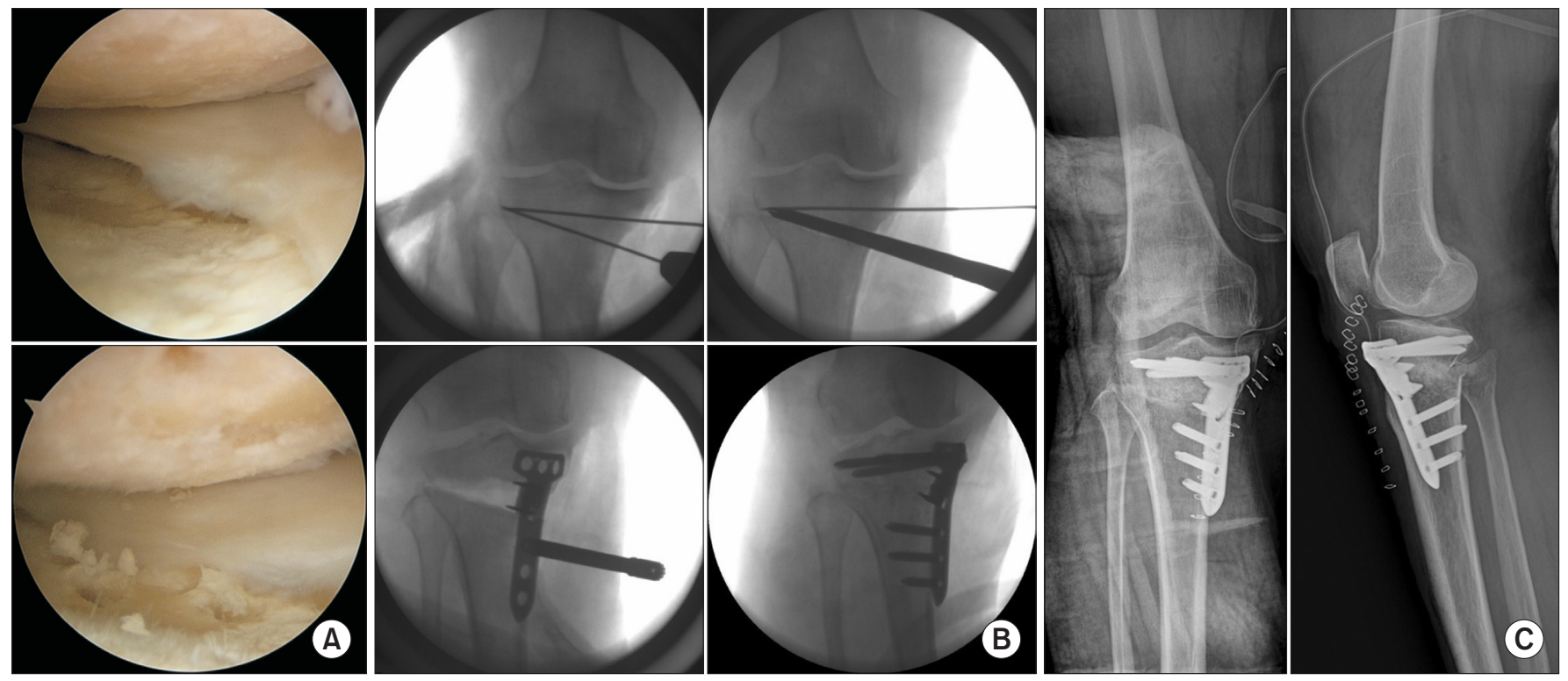

Fig. 1. (A) Arthroscopy revealed cartilage lesions in the medial femoral condyle and medial tibial condyle, and microfracture was performed. (B) Intraoperative anteroposterior fluoroscopic radiographs. (C) Postoperative radiographs obtained after medial opening wedge high tibial osteotomy and fixation with a locking plate (OhtoFix).
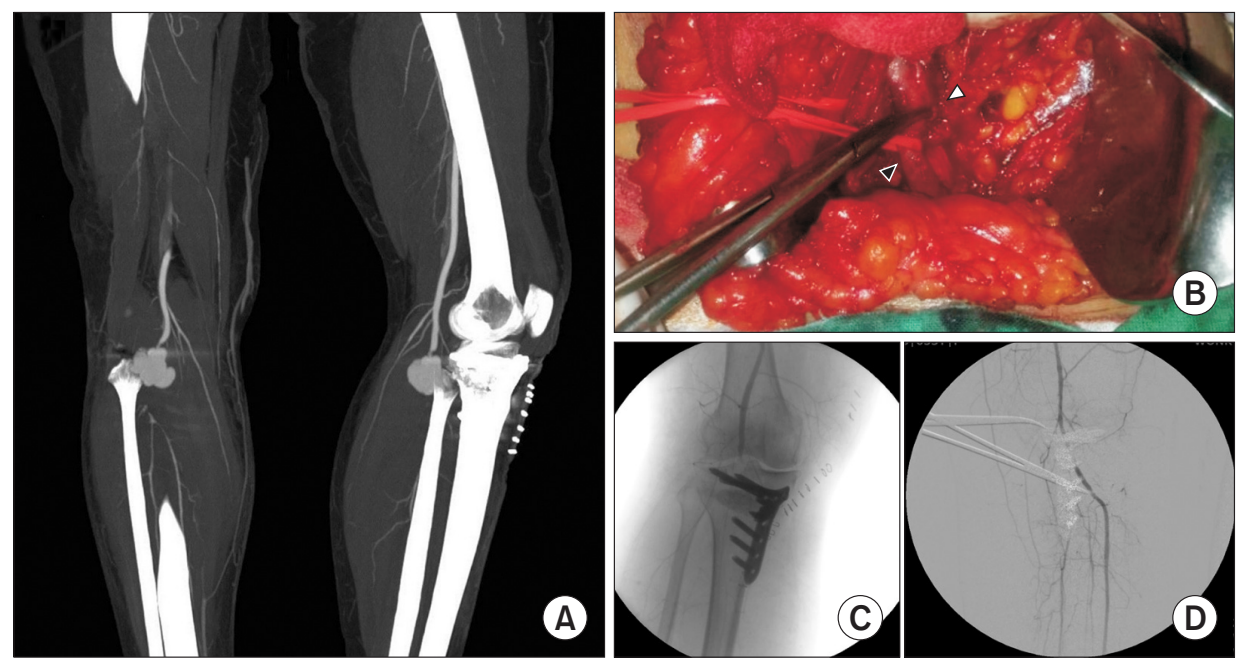

Fig. 2. (A) Vascular computed tomography angiography performed three days after surgery showing a pseudoaneurysm of the popliteal artery at the level of proximal screw fixation. (B) After hematoma evacuation, the popliteal vein (black arrowhead) and the proximal end of the ruptured popliteal artery (white arrowhead) were observed. (C) Angiography performed before anastomosis showing low visualization of the distal tibial artery. (D) After interposition graft, distal tibial artery visualization was significantly improved.

parallel to the posterior tibial slope in a direction from $3.5 \mathrm{~cm}$ distal to the medial joint line to $1.5 \mathrm{~cm}$ distal to the lateral joint line. The leg was held in a slight flexion position (about $30^{\circ}$ ) during the osteotomy, drilling, and screw fixation.

An oscillating saw was only used to cut the entry of the near cortex, and osteotomes were used for the rest of the procedure. To prevent a fracture of the lateral cortex, the osteotomy was stopped 5-10 mm before reaching the lateral cortex ${ }^{1)}$. A fulcrum was made to open the osteotomy site, and the gap was slowly widened by inserting 3-4 chisels. A bone spreader was inserted deep into the osteotomy site; during the spreading process, a lat- eral cortex fracture developed unintentionally. While maintaining the gap by inserting a metal block in the medial cortex, the locking plate (OhtoFix; OhtoMedical Co., Goyang, Korea) was used for fixation. The tourniquet was decompressed to confirm the presence of a vascular injury. Upon confirming that there was no prominent bleeding, bone grafting with bone chip allograft was performed to complete the operation (Fig. 1B and C).

Dorsalis pedis and posterior tibial pulses and ankle dorsiflexion were normal in the recovery room immediately after the operation. The patient was in a bed rest state and did not complain of any abnormalities until the second night of operation. On the 
third postoperative day, at 7:00 am, the patient complained of motor weakness (ankle dorsiflexion M1 and great toe extension M1) and paresthesia of the dorsum of the foot and web space between the great toe and the second toe. Compression bandage was removed immediately, and prominent swelling was observed in the popliteal fossa. Pulsation was felt inside the swelling site. Pallor or poikilothermia of the distal portion was not observed, but dorsalis pedis and posterior tibial pulses were not palpable. It was suspected that compartment syndrome and peroneal nerve palsy symptoms occurred due to a vascular injury. An emergency vascular computed tomography (CT) revealed a pseudoaneurysm due to popliteal artery tearing, which was surrounded by the gastrocnemius muscle. There was a contrast filling defect due to a thrombus but the distal flow was partially preserved (Fig. 2A).

Emergency fasciotomy and hematoma evacuation and exploration were done immediately in cooperation with vascular surgeons. The popliteal artery was separated and the proximal end was located at the mid popliteal fossa (Fig. 2B). The distal arterial stump was embedded in the interosseous membrane. The proximal and distal stumps were dissected carefully for anastomosis. The ipsilateral great saphenous vein at the knee joint level was harvested for interposition graft. Distal and proximal anastomosis was done with 7-0 prolene. After surgery, visualization of the distal tibial artery was significantly improved on CT angiography (Fig. 2C and D).

We retrospectively reviewed the preoperative MRI and postoperative CT to determine the cause of popliteal artery pseudoaneurysm. There was no proximal screw protruding the far cortex on the postoperative CT. However, another drilled hole with no inserted screw was heading the popliteal artery and it was confirmed that it was extending beyond the posterior cortex (Fig. $3 \mathrm{~A}-\mathrm{C}$ ). After drilling of the proximal hole and locking screw insertion, the plate had not been aligned with the bone contour, so we repositioned the plate after screw removal. Compared with the position of the popliteal artery at the same level on the preoperative MRI, it was found to be very close to the drilled hole of the posterior cortex; thus, we conjectured it was caused by drilling.

CT angiography at 8 months postoperatively showed that blood flow was maintained without obstruction of the graft site (Fig. $4 \mathrm{~A})$. Dorsiflexion of the ankle was gradually recovered to grade M3, but paresthesia symptoms remained in the dorsum of the foot. There was no pain at the operation site, and walking was
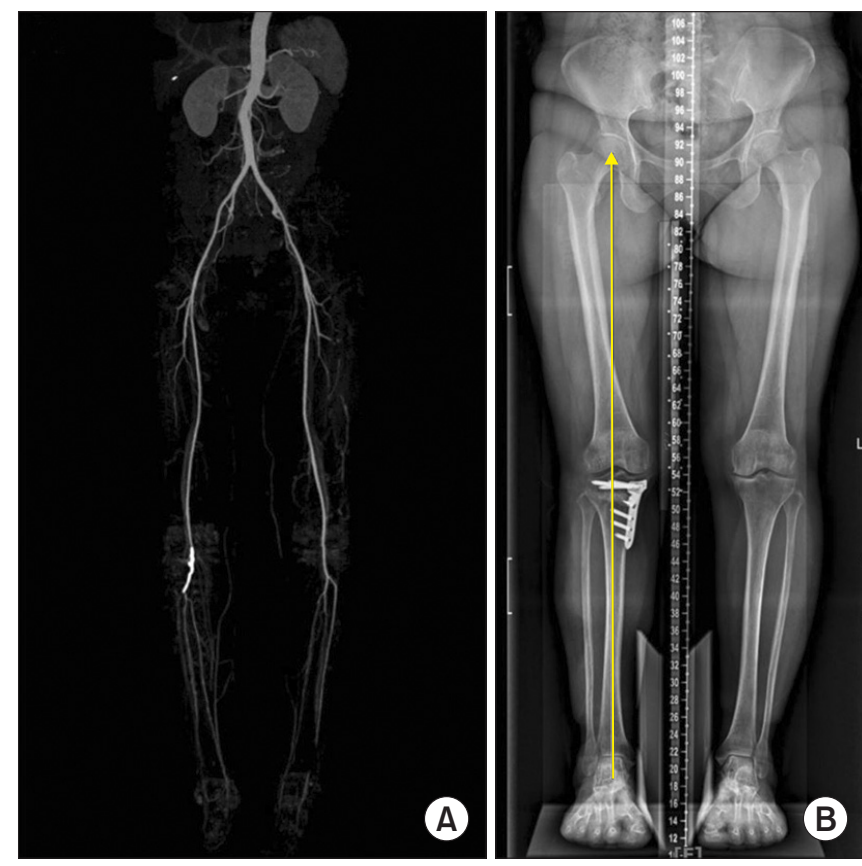

Fig. 4. Postoperative 8-month vascular computed tomography (CT) angiography and radiography. (A) Vascular CT angiogram showing blood flow was maintained without obstruction of the graft site. (B) Long-leg standing radiograph showing the mechanical axis of the lower limb (arrow) was maintained without reduction of the correction angle.
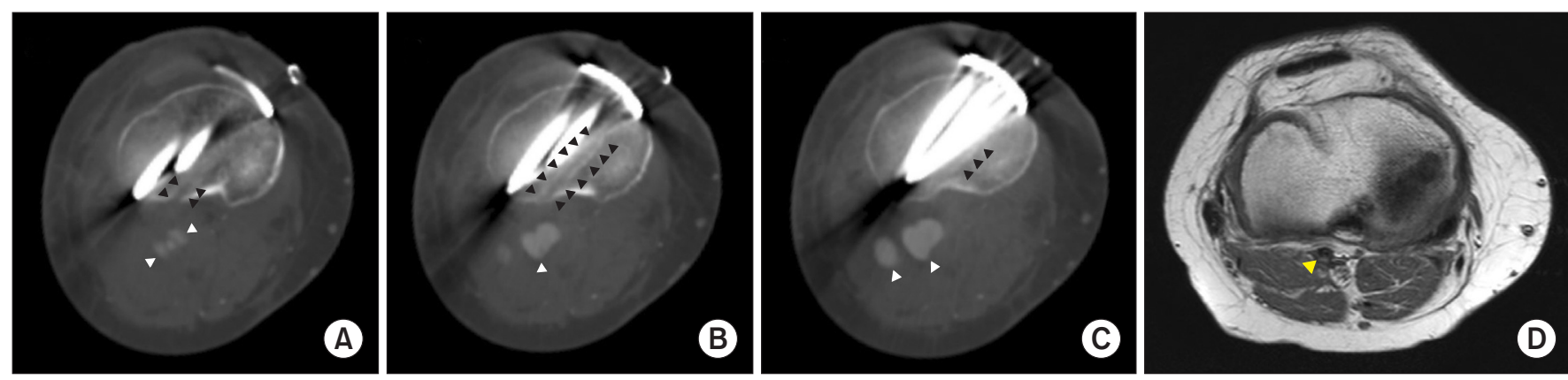

Fig. 3. (A-C) Consecutive three cuts of the axial view of the proximal screw portion of the vascular computed tomography performed three days after surgery. The direction of the drill was slightly upward, penetrating the posterior cortex (black arrowhead). Pseudoaneurysm was formed in the adjacent region (white arrowhead). (D) The location of the popliteal artery on preoperative magnetic resonance imaging (arrowhead). 
possible using a cane. There was no evidence of nonunion of the bone graft site on the radiographs and the mechanical axis of the lower limb was maintained without reduction of the correction angle (Fig. 4B).

\section{Discussion}

Injury of the popliteal and anterior tibial arteries is a rare complication of $\mathrm{HTO}^{2-4)}$. In addition, most of the injuries of the popliteal and anterior tibial arteries occur during lateral close wedge $\mathrm{HTO}^{2}$. Several cases of vascular injuries were reported following MOWHTO, which was due to the use of an osteotome ${ }^{3,4)}$ or an oscillating saw for tibial osteotomy ${ }^{5)}$. However, there has been no report of injury of the popliteal artery due to drilling during screw fixation of the locking plate after the osteotomy.

The popliteal artery is farthest from the posterior tibia at $90^{\circ}$ of knee flexion, and it is generally recommended that osteotomy should be performed at $90^{\circ}$ knee flexion position ${ }^{3,6,7)}$. However, this method does not guarantee complete protection, since rupture of the dorsal artery may occur even at sufficient knee flexion position above $90^{\circ 3,5)}$. Kim et al. ${ }^{6)}$ recommended that the saw angle be kept as flat as possible; different saw angles were compared against the coronal plane in the cadaver knees and angles greater than $30^{\circ}$ could cause damage to the dorsal arteries. Tourniquet pressure was released to control the bleeding before wound closure. Severe bleeding should be suspected of vascular damage, and diagnosis should be done immediately. Therefore, it is advisable to decompress the tourniquet before wound closure to evaluate the amount of bleeding ${ }^{3)}$. Bernhoff and Bjorck ${ }^{4)}$ analyzed 21 iatrogenic popliteal artery injuries during non-arthroplasty knee surgery. Nine injuries were detected intraoperatively, five within 12-48 hours and seven $>48$ hours postoperatively. In addition, the prognosis was poorer when there was a delay of diagnosis and treatment. In this patient, popliteal arterial injury was found within 3 days after surgery even though there was no hemorrhage after tourniquet decompression after osteotomy. However, rapid vascular surgery was performed after the diagnosis, and fortunately, the results were relatively good.

Pseudoaneurysm arises from a disruption in the continuity of the arterial wall. The effect of arterial pressure on arterial pressure creates a perfused sac that communicates with the arterial lumen. The perfused sac is simply contained by the media or adventitia or simply by soft tissue structures surrounding the damaged ves$\mathrm{sel}^{8)}$. A localized audible bruit or pulsating mass may be present. Ischemia of the surrounding tissues due to vascular compromise may result in necrosis of the overlying skin. Neurological symp- toms may develop secondary to nerve compression or ischemia. Duplex ultrasonography, CT angiography, and MRI are useful for diagnosis and treatment planning ${ }^{9)}$. Depending on the nature of the pseudoaneurysm, various treatments such as coiling, thrombin injection, end-to-end anastomosis, graft placement, and bypass may be selected ${ }^{4,10)}$. In our patient, the pseudoaneurysmal cyst was already torn and the proximal and distal portions were separated when the popliteal artery was exposed, and saphenous vein interposition graft was selected as the treatment method.

It is important to note that the occurrence of neurovascular injury during MOWHTO can lead to fatal outcomes. In order to minimize posterior neurovascular injury, the osteotomy site should be carefully selected. In addition, it is preferable not to drill the far cortex in the proximal part of the osteotome site when a drill bit is used, and the position of the metal plate should be positioned posteromedially as much as possible. Furthermore, it is important to check for bleeding after tourniquet decompression to confirm the absence of a vascular injury, but pseudoaneurysms that may occur with delayed presentation should not be overlooked.

\section{Conflict of Interest}

No potential conflict of interest relevant to this article was reported.

\section{References}

1. Han SB, Lee DH, Shetty GM, Chae DJ, Song JG, Nha KW. A "safe zone" in medial open-wedge high tibia osteotomy to prevent lateral cortex fracture. Knee Surg Sports Traumatol Arthrosc. 2013;21:90-5.

2. Sawant MR, Ireland J. Pseudo-aneurysm of the anterior tibial artery complicating high tibial osteotomy: a case report. Knee. 2001;8:247-8.

3. Attinger MC, Behrend $\mathrm{H}$, Jost B. Complete rupture of the popliteal artery complicating high tibial osteotomy. J Orthop. 2014;11:192-6.

4. Bernhoff $\mathrm{K}$, Bjorck M. Iatrogenic popliteal artery injury in non arthroplasty knee surgery. Bone Joint J. 2015;97:192-6.

5. Georgoulis AD, Makris CA, Papageorgiou CD, Moebius UG, Xenakis T, Soucacos PN. Nerve and vessel injuries during high tibial osteotomy combined with distal fibular osteotomy: a clinically relevant anatomic study. Knee Surg Sports Traumatol Arthrosc. 1999;7:15-9.

6. Kim J, Allaire R, Harner CD. Vascular safety during high tib- 


\section{Chun et al. Pseudoaneurysm after OWHTO}

ial osteotomy: a cadaveric angiographic study. Am J Sports Med. 2010;38:810-5.

7. Yoo JH, Chang CB. The location of the popliteal artery in extension and 90 degree knee flexion measured on MRI. Knee. 2009;16:143-8.

8. Saad NE, Saad WE, Davies MG, Waldman DL, Fultz PJ, Rubens DJ. Pseudoaneurysms and the role of minimally invasive techniques in their management. Radiographics. 2005;
25 Suppl 1:S173-89.

9. Sigterman TA, Dolmans DE, Welten RJ, Krasznai A, Bouwman LH. Anterior tibial artery aneurysm: case report and literature review. Int J Surg Case Rep. 2013;4:243-5.

10. Boologapandian V, Joseph A, Selvapackiam J, Narayanan S, Paramasivam I. Anterior tibial artery pseudoaneurysm: case series. Indian J Vasc Endovasc Surg. 2017;4:112-4. 